\title{
Non-Markovian diffusion over a parabolic potential barrier: influence of the friction-memory function
}

\author{
B. Yilmaz ${ }^{1,2}$, S. Ayik ${ }^{3}$, Y. Abe ${ }^{4}$, and D. Boilley ${ }^{5}$ \\ ${ }^{1}$ Physics Department, Ankara University, 06100 Ankara, Turkey \\ ${ }^{2}$ Physics Department, Middle East Technical University, 06531 Ankara, Turkey \\ ${ }^{3}$ Physics Department, Tennessee Technological University, Cookeville, TN 38505, USA \\ ${ }^{4}$ Research Center for Nuclear Physics, \\ Osaka University, Osaka, Japan and \\ ${ }^{5}$ Grand Accélérateur National d'Ions Lourds (GANIL), \\ CEA/DSM-CNRS/IN2P3, BP 55027, F-14076 Caen Cedex 5, France
}

(Dated: November 23, 2018)

\begin{abstract}
The over-passing probability across an inverted parabolic potential barrier is investigated according to the classical and quantal generalized Langevin equations. It is shown that, in the classical case, the asymptotic value of the over-passing probability is determined by a single dominant root of the characteristic function, and it is given by a simple expression. The expression for the overpassing probability is quite general, and details of dissipation mechanism and memory effects enter into the expression only through the dominant root of the characteristic equation.

PACS numbers: 05.40.-a, 66.10.C-
\end{abstract}




\section{INTRODUCTION}

In many physical systems, for example transport processes in condensed matter physics, activation processes in chemical reactions, and thermal fission and fusion reactions in nuclear physics, generalized Langevin approach provides a very useful framework for theoretical description of the reaction under consideration [1, 2, 3, 4, 5, 6, 7]. According to general framework of Mori [8], the equations of motion of relevant variables, in general, appear as non-Markovian stochastic differential equations, referred to as Generalized Langevin Equations (GLE). These equations involve memory dependent dissipation and correlated random forces, which are connected to each other in accordance with the fluctuation-dissipation relation of non-equilibrium statistical mechanics. It is possible to derive the GLE in the classical limit, and also including quantum statistical effects [9, 10, 11, 12, 13, 14]. The generalized Langevin approach has recently gained a lot of interest as a mathematical tool to deal with diffusion in disordered medium, phenomenon known as anomalous diffusion which is characterized by a long-range power-law correlations encountered in various physical processes

such as the dynamics of polymers [15], decorrelation processes in microemulsions [16], charge transport in amorphous semiconductors [17], and diffusion in fractals [18].

After the pioneering work of Kramers, the Langevin approach has been applied to describe normal as well as anomalous diffusion over a potential barrier in many research subjects. In order to solve the GLE, one must assume a particular form for the spectral density of the environment or the memory kernel which define the non-Markovian effects. In our study, we investigate the consequences of the non-Markovian effects on the asymptotic behavior of the system. We consider the evolution of a single-relevant variable with sharp initial values according to the classical and quantal GLE. The noise term in the GLE is a Gaussian stochastic variable and hence the probability distribution has a Gaussian form, which is specified by the mean-values and the variances of the relevant variables. In the specific case of exponential Friction-Memory Function (FMF), the non-Markovian problem was solved analytically in [19]. Here, we consider a general form of the FMF, and investigate memory effects on the dynamical evolution of normal as well as anomalous systems. We show that for classical GLE the asymptotic value of the over-passing probability is determined by a single dominant root of the characteristic function Eq. (86) and given by a simple expression Eq. (15). This expression for the over-passing probability is quite general, and details 
of dissipation mechanism and memory effects enter into the expression only through the dominant root of the characteristic equation [19]. In the case of quantal GLE, the asymptotic expression of the over-passing probability has the same structure as the classical case, except it involves a quantity which is determined by a numerical integration over the spectral density.

The formal expression for the over-passing probability is derived in Section II. The analysis of the probability and some general results are explained in Section III. The conclusion is given in Section IV.

\section{FORMAL EXPRESSION FOR THE OVER-PASSING PROBABILITY}

\section{A. The over-passing probability for the classical GLE}

The classical GLE reads

$$
\ddot{q}(t)=-\frac{1}{m} \frac{\partial V}{\partial q}-\int_{0}^{t} \chi\left(t-t^{\prime}\right) \dot{q}\left(t^{\prime}\right) d t^{\prime}+\epsilon(t),
$$

where $\chi(t)$ is the model dependent FMF and the stochastic driving term $\epsilon(t)$ has a Gaussian distribution with first and second moments given by

$$
\begin{aligned}
\langle\epsilon(t)\rangle & =0, \\
\left\langle\epsilon(t) \epsilon\left(t^{\prime}\right)\right\rangle & =\frac{T}{m} \chi\left(\left|t-t^{\prime}\right|\right),
\end{aligned}
$$

so that the classical fluctuation-dissipation theorem is satisfied. Here, $T$ is the temperature of the heat-bath. All throughout the paper, we set $k_{B}=1$ where $k_{B}$ is the Boltzmann constant.

For a quadratic potential barrier with a barrier height $B$ which is defined by the initial position $q_{0}$ as

$$
V(q)=\frac{1}{2} m \Omega^{2}\left(q_{0}^{2}-q^{2}\right)=B-\frac{1}{2} m \Omega^{2} q^{2}
$$

using the Laplace transform of Eq. (1), the mean and the variance of $q(t)$ over the noise, denoted by $\langle.$.$\rangle , can be obtained as$

$$
\langle q(t)\rangle=q_{0}\left[1+\Omega^{2} \int_{0}^{t} h\left(t^{\prime}\right) d t^{\prime}\right]+\frac{p_{0}}{m} h(t),
$$


and

$$
\sigma_{q q}(t)=\frac{T}{m} \int_{0}^{t} d t^{\prime} \int_{0}^{t} d t^{\prime \prime} h\left(t^{\prime}\right) h\left(t^{\prime \prime}\right) \chi\left(\left|t^{\prime}-t^{\prime \prime}\right|\right)
$$

respectively. The time-dependent function $h(t)$ reads

$$
\begin{aligned}
h(t) & =\mathcal{L}^{-1}[1 / D(s)] \\
& =\sum_{i} \operatorname{Res}\left[\tilde{h}\left(s_{i}\right)\right] e^{s_{i} t},
\end{aligned}
$$

where $\mathcal{L}^{-1}$ stands for inverse Laplace transform and $\operatorname{Res}\left[\tilde{h}\left(s_{i}\right)\right]$ is the residue of $\tilde{h}(s)=\mathcal{L}[h(t)]$ at the roots (poles) $s_{i}$ of the characteristic function

$$
D(s)=s^{2}+s \tilde{\chi}(s)-\Omega^{2}
$$

with $\tilde{\chi}(s)=\mathcal{L}[\chi(t)]=\int_{0}^{\infty} \exp (-s t) \chi(t) d t$ being the Laplace transform of $\chi(t)$. The initial position $q_{0}$ as well as the initial momentum $p_{0}$ of the collective system are considered to be sharp. The formal expressions for $\langle p(t)\rangle, \sigma_{p p}(t)$ and $\sigma_{q p}(t)$ can also be obtained, but are irrelevant for finding the over-passing probability. For a quadratic potential, the relevant variables have Gaussian distribution. By integrating out the momentum, the reduced distribution

$$
W(q)=\frac{1}{\sqrt{2 \pi \sigma_{q q}(t)}} \exp \left(-\frac{(q-\langle q(t)\rangle)^{2}}{2 \sigma_{q q}(t)}\right)
$$

is obtained. Starting with the initial value $q_{0}<0$, the over-passing probability is simply the probability that the system is found on the other side of the potential barrier, hence the probability reads [20, 21],

$$
\begin{aligned}
P(t) & =\int_{0}^{\infty} W(q) d q \\
& =\frac{1}{2} \operatorname{Erfc}\left\{-\frac{\langle q(t)\rangle}{\sqrt{2 \sigma_{q q}(t)}}\right\} .
\end{aligned}
$$

This converges to a finite value,

$$
P=P(t \rightarrow \infty)=\frac{1}{2} \operatorname{Erfc}\left\{-\frac{\langle q(t \rightarrow \infty)\rangle}{\sqrt{2 \sigma_{q q}(t \rightarrow \infty)}}\right\}
$$

which defines the asymptotic value of the over-passing probability.

In various studies on activated rate processes the characteristic function Eq. (8) appears [4, 22, 23, 24, 25, 26, 27, 28] and it is shown that Eq. (8) has only one positive root (or pole), 
called hereafter $s_{1}$, which is larger than the real parts of all the others, see the appendix of [25] for details. This suggests that the asymptotic behavior of Eq. (7) is

$$
h(t \rightarrow \infty)=\operatorname{Res}\left[\tilde{h}\left(s_{1}\right)\right] e^{s_{1} t} .
$$

Therefore using Eq. (12), the Eqs. (15) and (6) read

$$
\langle q(t \rightarrow \infty)\rangle=\operatorname{Res}\left[\tilde{h}\left(s_{1}\right)\right]\left(\frac{q_{0} \Omega^{2}}{s_{1}}+\frac{p_{0}}{m}\right) e^{s_{1} t},
$$

and

$$
\sigma_{q q}(t \rightarrow \infty)=\left(\operatorname{Res}\left[\tilde{h}\left(s_{1}\right)\right]\right)^{2} \frac{T}{m}\left(\frac{\Omega^{2}-s_{1}^{2}}{s_{1}^{2}}\right) e^{2 s_{1} t}
$$

where the last equation is obtained by performing the integration of Eq. (6) using the variables $u=t^{\prime}+t^{\prime \prime}$ and $v=t^{\prime}-t^{\prime \prime}$ and then using the equation $D\left(s_{1}\right)=0$. Substituting Eqs. (13) and (14) into Eq. (11) we get the over-passing probability in the form

$$
P=\frac{1}{2} \operatorname{Erfc}\left\{\frac{1}{\sqrt{1-y^{2}}}\left(\sqrt{\frac{B}{T}}-y \sqrt{\frac{K}{T}}\right)\right\},
$$

where $B$ is the barrier height measured from the initial position defined in Eq. (4) whereas $K=p_{0}^{2} / 2 m$ is the initial kinetic energy. The function $y$ is given by

$$
y=\frac{s_{1}}{\Omega} .
$$

If $p_{0}>0$, there exists a critical initial kinetic energy $K_{c}=B / y^{2}$ for which the mean trajectory Eq. (13) converges to the top of the barrier, $\langle q(t \rightarrow \infty)\rangle=0$ and hence the kernel of the error function in Eq. (15) vanishes to give the probability $P=1 / 2$. Since the critical kinetic energy must be larger than the barrier height $B$, the function $y$ can assume any value in the interval,

$$
0 \leq y \leq 1
$$

The function $y$ is the Kramers factor in the rate formula for a non-Markovian escape process from a metastable state [4, 22, 23, 24, 25, 26, 27, 29] and here it is the function that determines the non-Markovian effects on the over-passing probability. Figure 1 shows the probability, Eq. (15), being plotted versus $y$ for three energy regions. For $y=1$, the probability takes its classical value, which correspond to the trivial system without 


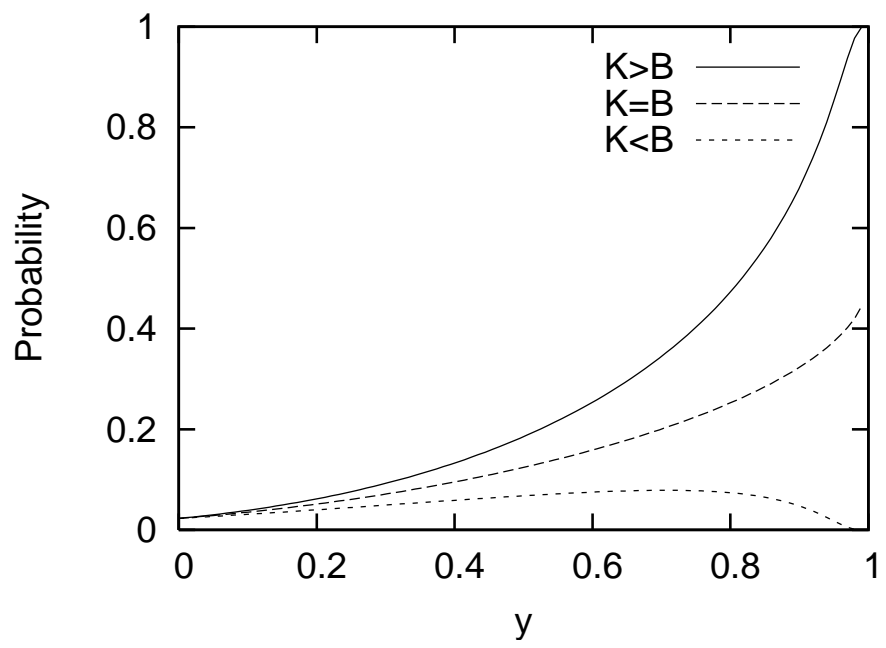

FIG. 1: The asymptotic probability, Eq. (15), is plotted versus the parameter $y$ for the three energy regions. The temperature is taken such that $T / B=0.5$ and the solid, dashed and dotted lines stand for $K / B=1.5,1,0.5$ values, respectively.

dissipation. The probability for the over-damped system is obtained for $y=0$, which is the smallest probability for kinetic energies $K$ larger or equal to the barrier height $B$. Then, the function $y$ can be termed as "the dissipation reducing factor". However, when the kinetic energy is smaller than the barrier height we have a more interesting situation where the maximum

$$
P_{\max }=\frac{1}{2} \operatorname{Erfc}\left\{\sqrt{\frac{B-K}{T}}\right\} \quad K \leq B,
$$

occurs at some mid-point value

$$
y_{\max }=\sqrt{\frac{K}{B}} \quad K \leq B .
$$

Since the function $y$ is the only positive root $s_{1}$ of Eq. (8) divided by the curvature parameter $\Omega$ of the potential barrier, it depends on the specific form of the FMF and is a function of the parameters that FMF is expressed by as well as the curvature parameter $\Omega$ of the potential. There will be a specific set of these parameters for which $y_{\max }$ in Eq. (19) will be obtained. The probability $P_{\max }$ occurs due to the compensation between dissipation which reduces probability and fluctuation which enhances probability. This is explained in the next section.

It should be emphasized here that the results Eq. (15) and Eq. (18) are valid for any FMF $\chi(t)$ whose Laplace transform exists. The formal simplicity of these expressions is due 
to the fact that the asymptotic behavior $(t \rightarrow \infty)$ of non-Markovian systems with Gaussian noises diffusing over a parabolic barrier can be reduced to that of Markovian ones with an effective friction coefficient,

$$
\beta_{\mathrm{eff}}=\tilde{\chi}\left(s_{1}\right)
$$

which contains all non-Markovian effects. This is easily seen when the normalized root, using Eq. (8), is cast into the formal form,

$$
y=\sqrt{1+\left(\frac{\beta_{\mathrm{eff}}}{2 \Omega}\right)^{2}}-\frac{\beta_{\mathrm{eff}}}{2 \Omega},
$$

which has the same form with the Markovian factor Eq. (28).

\section{B. The over-passing probability for the quantum GLE}

For systems with quadratic potentials, the difference between the classical and c-number quantum GLE is the correlation of the stochastic force [1, 2, 30], hence the quantum GLE has the same form with Eq. (1) but with a mean-zero Gaussian noise satisfying the correlation

$$
\left\langle\epsilon(t) \epsilon\left(t^{\prime}\right)\right\rangle=\frac{1}{m} \int_{-\infty}^{\infty} \frac{d \omega}{\pi} T^{\star}(\omega) \hat{\chi}_{\text {real }}(\omega) e^{-i \omega\left(t-t^{\prime}\right)}
$$

instead of Eq. (3). $T^{\star}$ is the effective temperature given by

$$
T^{\star}(\omega)=\frac{\hbar \omega}{2} \operatorname{coth}\left(\frac{\hbar \omega}{2 T}\right),
$$

and $\hat{\chi}_{\text {real }}(\omega)$ is the real part of the Fourier transformed FMF $\hat{\chi}(\omega)=\int_{-\infty}^{\infty} \chi(t) \exp (i \omega t) d t$. The effective temperature is the mean energy of a quantum harmonic oscillator and for high temperatures $\hbar \omega \ll 2 T$, it takes its classical value $T^{\star} \rightarrow T$. The full quantum limit $T^{\star} \rightarrow$ $\hbar \omega / 2$ is obtained at low temperatures $\hbar \omega \gg 2 T$ and represents the zero-point (vacuum) energy.

Since the noise term does not appear in the expression of the mean position, the Eqs. (5) and (13) are valid for quantum systems as well, whereas the variance of the position takes the form

$$
\begin{aligned}
\sigma_{q q}(t)= & \frac{1}{m} \int_{0}^{t} d t^{\prime} \int_{0}^{t} d t^{\prime \prime} h\left(t^{\prime}\right) h\left(t^{\prime \prime}\right) \\
& \times \int_{-\infty}^{\infty} \frac{d \omega}{\pi} T^{\star}(\omega) \hat{\chi}_{\text {real }}(\omega) e^{-i \omega\left(t^{\prime}-t^{\prime \prime}\right)}
\end{aligned}
$$


By using Eq. (12), the asymptotic value of the variance reads

$$
\begin{aligned}
\sigma_{q q}(t \rightarrow \infty)= & \frac{1}{m}\left(\operatorname{Res}\left[\tilde{h}\left(s_{1}\right)\right]\right)^{2} e^{2 s_{1} t} \\
& \times \int_{-\infty}^{\infty} \frac{d \omega}{\pi} T^{\star}(\omega) \frac{\hat{\chi}_{\text {real }}(\omega)}{\omega^{2}+s_{1}^{2}} .
\end{aligned}
$$

Substituting Eq. (13) and Eq. (25) into Eq. (11), we get the over-passing probability as

$$
P=\frac{1}{2} \operatorname{Erfc}\left\{\frac{1}{\sqrt{G(y)}}(\sqrt{B}-y \sqrt{K})\right\},
$$

where

$$
G(y)=y^{2} \int_{-\infty}^{\infty} \frac{d \omega}{\pi} T^{\star}(\omega) \frac{\hat{\chi}_{\text {real }}(\omega)}{\omega^{2}+s_{1}^{2}} .
$$

\section{ANALYSIS OF THE PROBABILITY}

\section{A. Influence of the memory}

The knowledge of the FMF $\chi(t)$ is crucial for determining the over-passing probability since the probabilities Eq. (15) and Eq. (26) are functions of the positive root of the characteristic function Eq. (8) which depends on the Laplace transform $\tilde{\chi}(s)$. As an example, in the Markovian (M) limit, the normalized root $y$ is explicitly given by

$$
y^{(\mathrm{M})}=\sqrt{1+\left(\frac{\beta}{2 \Omega}\right)^{2}}-\frac{\beta}{2 \Omega}
$$

for the FMF [20],

$$
\chi^{(\mathrm{M})}(t)=2 \beta \delta(t),
$$

which corresponds to a memoryless dissipation with a reduced friction coefficient $\beta$. For non-Markovian Exponential (E) FMF [19],

$$
\chi^{(\mathrm{E})}(t)=\frac{\beta}{\tau} \exp \left(-\frac{t}{\tau}\right),
$$

the normalized root can be expressed as 


$$
\begin{aligned}
y^{(\mathrm{E})=} & -\frac{1}{3 \Omega \tau} \\
& -\frac{1}{3 \Omega \tau} \frac{-1+3 \beta \tau-3(\Omega \tau)^{2}}{\left[-1+\frac{9}{2} \beta \tau+9(\Omega \tau)^{2}+\sqrt{\left(-1+\frac{9}{2} \beta \tau+9(\Omega \tau)^{2}\right)^{2}+\left(-1+3 \beta \tau-3(\Omega \tau)^{2}\right)^{3}}\right]^{1 / 3}} \\
& +\frac{1}{3 \Omega \tau}\left[-1+\frac{9}{2} \beta \tau+9(\Omega \tau)^{2}+\sqrt{\left(-1+\frac{9}{2} \beta \tau+9(\Omega \tau)^{2}\right)^{2}+\left(-1+3 \beta \tau-3(\Omega \tau)^{2}\right)^{3}}\right]^{1 / 3}(31)
\end{aligned}
$$

The exponential FMF corresponds to a dissipating system with a reduced friction coefficient $\beta$ and a memory characterized by the relaxation time $\tau$. In the limit $\tau \rightarrow 0^{+}$the exponential FMF Eq. (30) reduces to the Markovian FMF Eq. (29).

The over-passing probability for classical systems with Markovian FMF Eq. (29) and the exponential FMF Eq. (30) is obtained by substituting Eq. (28) and Eq. (31) into Eq. (15), respectively. Figure 2 shows the probability for the Markovian system plotted versus the initial kinetic energy over potential barrier height for various friction coefficients. The intersection points with the maximum probability (solid line) corresponds to the specific initial kinetic energy and specific friction $\beta / \Omega$ in Eq. (28) for which the condition Eq. (19) is met. In Figure 3, it is seen that as friction $\beta / \Omega$ increases, the factors $y^{(\mathrm{M})}$ and $y^{(\mathrm{E})}$ are

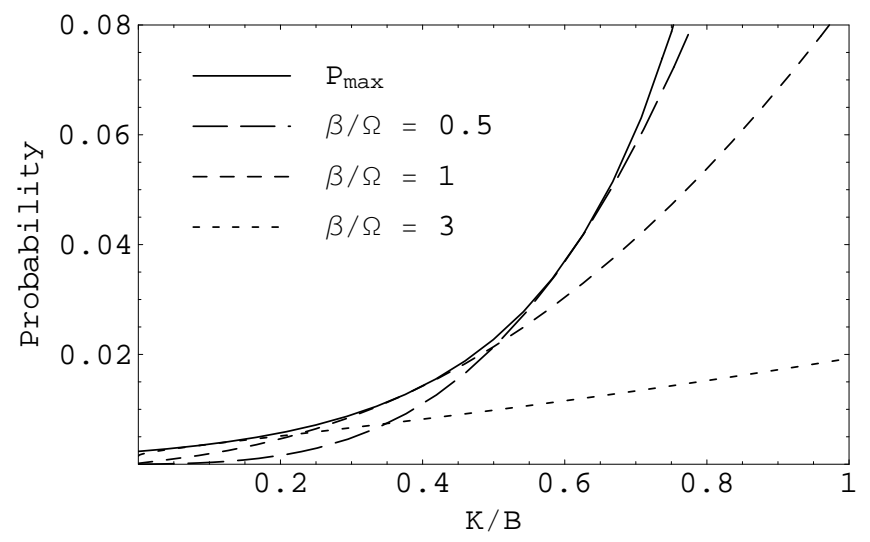

FIG. 2: The probability for classical Markovian case is plotted versus the initial kinetic energy over potential barrier height for various friction coefficients. The maximum probability, Eq. (18), is indicated by a solid line. The temperature is such that $T / B=0.25$.

decreasing. Increasing memory time $\Omega \tau$ results in increasing $y$ for any $\beta / \Omega$. Therefore by looking at the behavior of the probability with respect to the factor $y$ (see Figure 1), the 
friction $\beta / \Omega$ and memory time $\Omega \tau$ dependence of the probability is expected as in Figure 4 . For kinetic energies $K$ larger or equal to the barrier height $B$, the probability is decreasing as $\beta / \Omega$ is increasing which is due to the dissipation of kinetic energy. For zero kinetic energy the situation is opposite, as $\beta / \Omega$ is getting larger the probability is increasing which is due to the thermal fluctuations of the observables. In the intermediate region where the kinetic energy is less than the barrier height $B$, the probability obtains a peaked value, Eq. (18). This can be understood as the dissipation dominating at the right side of the maximum and fluctuation dominating at the left side of the maximum. In this region, the probability approaches the asymptotic value $0.5 \operatorname{Erfc}\{\sqrt{B / T}\}$ for the over-damped case $\beta / \Omega \rightarrow \infty$ regardless of the initial kinetic energy $K$ and memory time $\Omega \tau$.

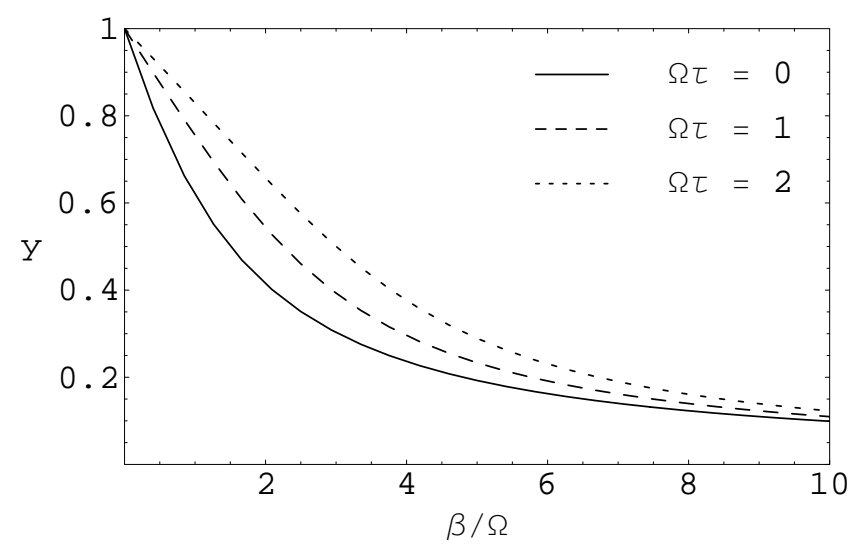

FIG. 3: The functions Eq. (28) (solid line) and Eq. (31) are plotted versus the friction $\beta / \Omega$ for different relaxation times $\Omega \tau$.

In general, by using Eq. (8) a relation between the root $y$ for any non-Markovian FMF $\chi(t)$ and the Markovian root $y^{(\mathrm{M})}$ given by Eq. (28) can be obtained as

$$
\frac{y}{y^{(\mathrm{M})}}=\frac{y^{(\mathrm{M})}+\beta^{(0)} / \Omega}{y+\beta_{\mathrm{eff}} / \Omega} \text {. }
$$

Here, the zero-frequency component of the Laplace transformed FMF defines the static friction,

$$
\beta^{(0)}=\tilde{\chi}(0)=\int_{0}^{\infty} \chi(t) d t
$$

which is equal to the friction coefficient $\beta$ in case of normal (non-anomalous) diffusion and $\beta_{\text {eff }}$ is the non-Markovian effective friction defined in Eq.(20). From Eq. (32) and Eq. (33), when 

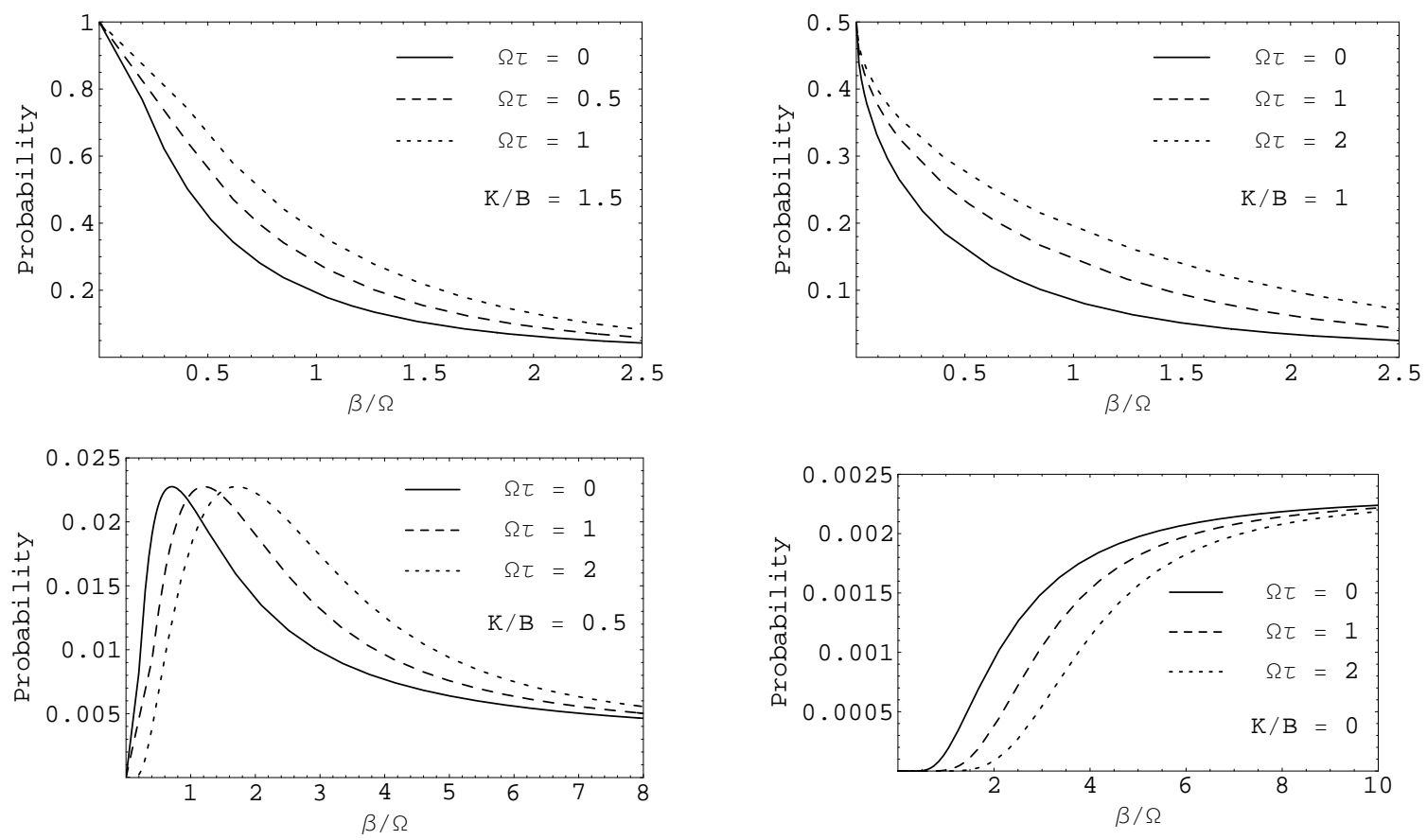

FIG. 4: The probability Eq. (15) for the exponential FMF Eq. (30) with the corresponding normalized positive root Eq. (31) is plotted versus the friction $\beta / \Omega$ for various memory times $\Omega \tau$. No memory case $\Omega \tau=0$ (solid lines) corresponds to the Markovian FMF Eq. (29) with $y$ given by Eq. (28). The temperature is taken so that $T / B=0.25$ and each figure is plotted with different initial kinetic energy $K / B=1.5,1,0.5,0$ as labeled on them.

$y$ is larger (smaller) than $y^{(\mathrm{M})}$, the non-Markovian effective friction $\beta_{\text {eff }}$ is smaller (larger) than the Markovian friction $\beta$ [22]. Hence by comparing the non-Markovian roots with Markovian roots, it is possible to relate the effective frictions and hence the probabilities.

\section{B. Influence of the oscillations}

It is possible to obtain an exact FMF for a system coupled to a heat-bath of harmonic oscillators known as Caldeira-Leggett Model [7, 31, 32, 33]. By using this model, the global degrees of freedom are reduced to the relevant ones and a GLE in the form of Eq. (11) is obtained. The corresponding FMF is a sum of cosine functions and hence has an oscillatory behavior. In order to understand the consequences of the oscillatory memories, let us 
consider the following FMF,

$$
\chi^{(\mathrm{EC})}(t)=\frac{\left(1+\lambda^{2}\right) \beta}{\tau} \exp \left(-\frac{t}{\tau}\right) \cos \left(\lambda \frac{t}{\tau}\right),
$$

where the parameter $\lambda$ keeps track of the oscillations (see Figure 5). The plots of the

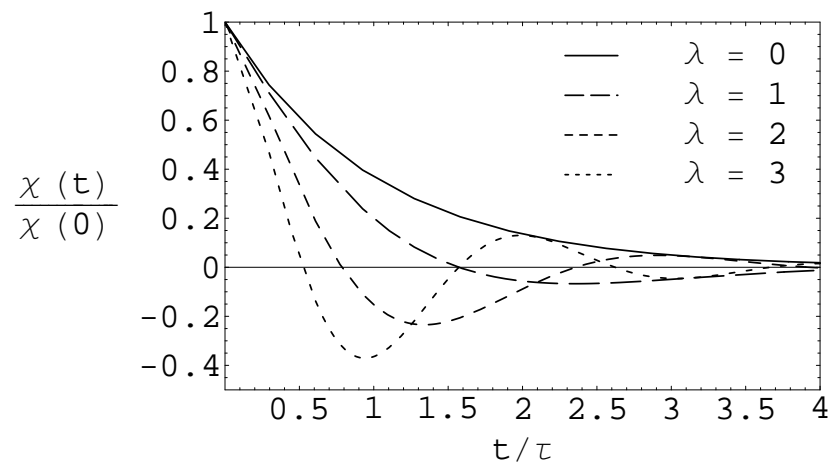

FIG. 5: The normalized FMF Eq. (34) is plotted versus time. Different lines correspond to different oscillation frequencies $\lambda$, the solid line with $\lambda=0$ is the exponential FMF.

corresponding normalized positive root $y^{(\mathrm{EC})}$ where EC stands for Exponential-Cosine FMF Eq. (34) and the corresponding over-passing probability for classical systems are shown in Figure 6 and Figure [7, respectively. It is seen that for small values of $\lambda$ the factor $y^{(\mathrm{EC})}$ is larger than $y^{(\mathrm{M})}$ (solid line) and for large values of $\lambda$ the factor $y^{(\mathrm{EC})}$ is smaller than $y^{(\mathrm{M})}$. By using the equation $\tilde{\chi}^{(\mathrm{EC})}\left(s_{1}\right)=\tilde{\chi}^{(\mathrm{EC})}(0)$ and the Laplace transform of Eq. (34), the critical value $\lambda_{c}$ for which $y^{(\mathrm{EC})}=y^{(\mathrm{M})}$ is satisfied can be found as

$$
\lambda_{c}=\sqrt{1+\Omega \tau y^{(\mathrm{M})}}
$$

which is in the interval $1<\lambda_{c}<\sqrt{1+\Omega \tau}$. When the dimensionless oscillation frequency is less than the critical value $\lambda<\lambda_{c}$, one has $y^{(\mathrm{EC})}>y^{(\mathrm{M})}$ and hence non-Markovian dissipation is smaller than the Markovian dissipation, $\beta_{\mathrm{eff}}<\beta$. This means that the oscillations of the FMF are irrelevant during the memory time $\Omega \tau$ and the dissipation is reduced like in the exponential FMF case. When the oscillations are relevant $\lambda>\lambda_{c}$, one has $y^{(\mathrm{EC})}<y^{(\mathrm{M})}$ and consequently the oscillations of the FMF cancel out in the characteristic time interval $\Omega \tau$ increasing the non-Markovian effective friction $\tilde{\chi}^{(\mathrm{EC})}\left(s_{1}\right)$ with respect to the Markovian friction $\tilde{\chi}^{(\mathrm{EC})}(0)$. 


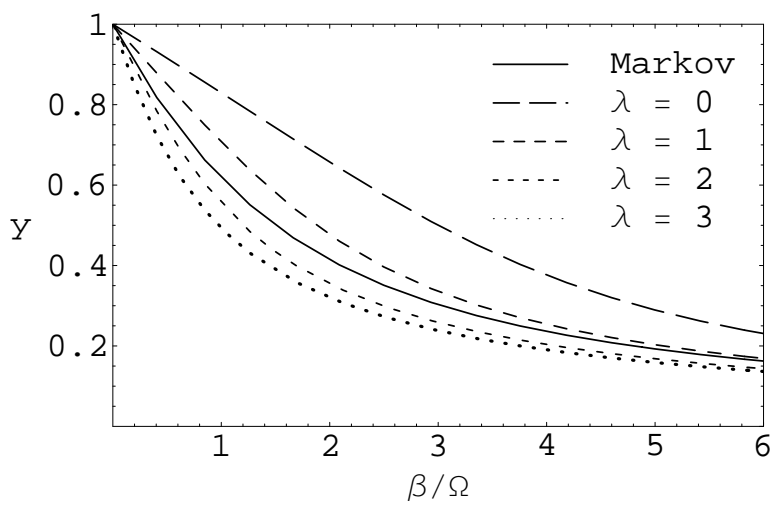

FIG. 6: The normalized positive root $y^{(\mathrm{EC})}$ of the Eq. (8) where the Laplace transform of Eq. (34) is used, is plotted versus friction $\beta / \Omega$ for various oscillation frequencies $\lambda$. The memory time is chosen as $\Omega \tau=2$. The solid line corresponds to the Markovian factor Eq. (28).

For the asymptotic value $\lambda \rightarrow \infty$, substituting the Laplace transform of Eq. (34) into Eq. (86) and taking the limit, the normalized positive root can be found as

$$
y_{\lambda \rightarrow \infty}^{(\mathrm{EC})}=\frac{1}{1+\beta \tau}\left[\sqrt{(1+\beta \tau)+\left(\frac{\beta}{2 \Omega}\right)^{2}}-\frac{\beta}{2 \Omega}\right],
$$

which satisfies the following inequality $y_{\lambda \rightarrow \infty}^{(\mathrm{EC})}<y_{\lambda>\lambda_{c}}^{(\mathrm{EC})}<y^{(\mathrm{M})}<y_{\lambda<\lambda_{c}}^{(\mathrm{EC})}$. The corresponding probabilities follow the same order, $P_{\lambda \rightarrow \infty}^{(\mathrm{EC})}<P_{\lambda>\lambda_{c}}^{(\mathrm{EC})}<P^{(\mathrm{M})}<P_{\lambda<\lambda_{c}}^{(\mathrm{EC})}$ for $K \geq B$.

\section{Influence of the anomalous diffusion}

The FMF for a system coupled to a Non-Ohmic (NO) heat-bath can be expressed as

$$
\chi^{(\mathrm{NO})}(t)=2 \int_{0}^{\infty} \frac{d \omega}{\pi} \frac{J(\omega)}{\omega} \cos (\omega t),
$$

where $J(\omega)$ is the spectral density of the heat bath. Non-Ohmic spectral density has the form [2, 5, 34, 35, 36, 37, 38, 39]

$$
J(\omega)=\beta \frac{\omega^{\alpha}}{\omega_{r}^{\alpha-1}} \quad(0<\alpha<2),
$$

where $\omega_{r}$ is some reference frequency allowing for consistent dimensionality of the friction $\beta$ for any $\alpha$. For matter of convenience, we set this frequency as that of the potential barrier, $\omega_{r}=\Omega$. The explicit form of the FMF is,

$$
\chi^{(\mathrm{NO})}(t)=\frac{2 \beta}{\pi \Omega^{\alpha-1}} \cos \left(\frac{\pi \alpha}{2}\right) \Gamma(\alpha) t^{-\alpha} \quad(t \neq 0),
$$



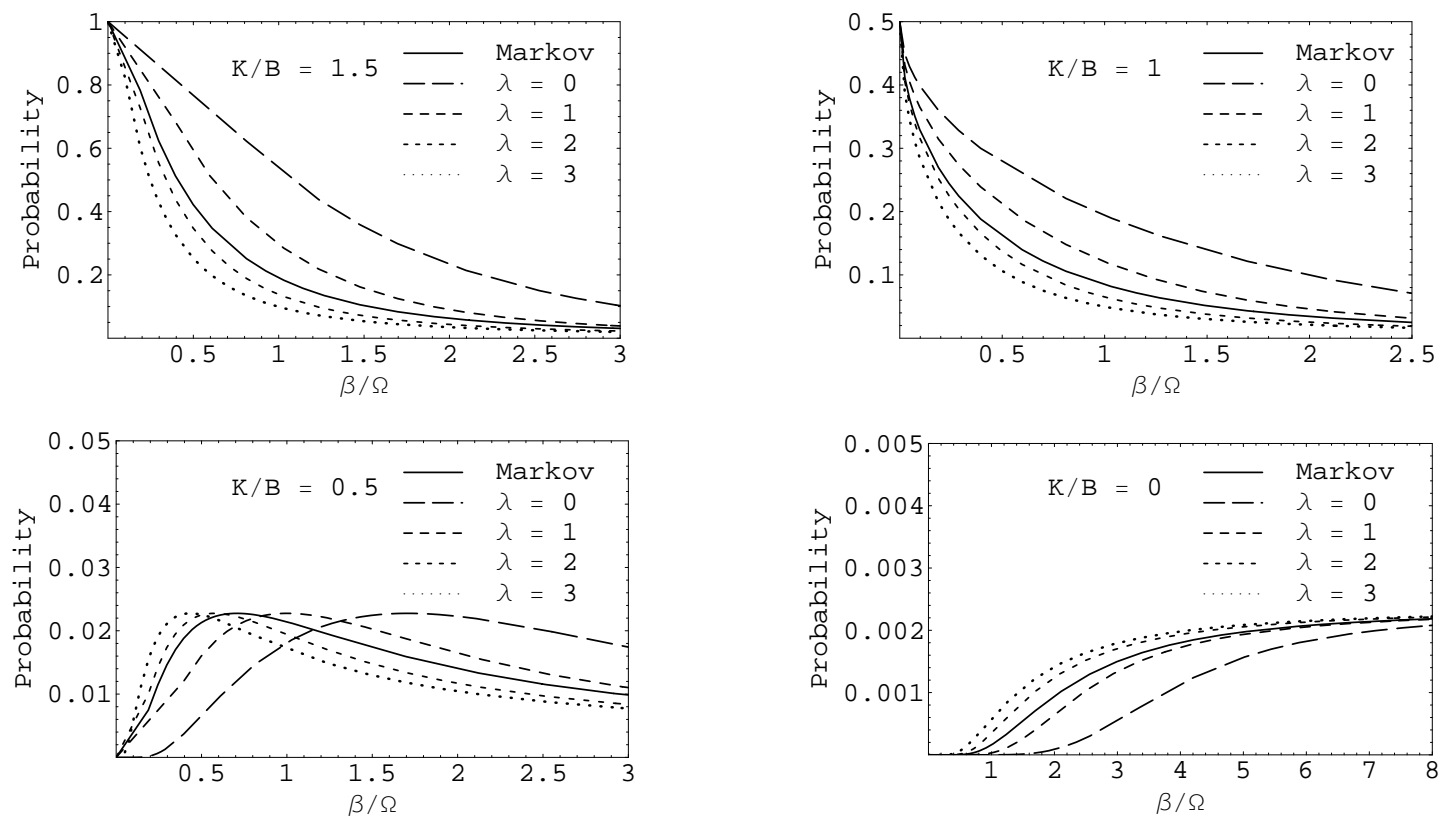

FIG. 7: The probability Eq. (15) for the exponential-cosine FMF Eq. (34) with the corresponding factor $y^{(\mathrm{EC})}$ shown in Figure 6, is plotted versus the friction $\beta / \Omega$ for various oscillation frequencies $\lambda$. The memory time is chosen as $\Omega \tau=2$. The Markovian probability is indicated by a solid line. The temperature is taken so that $T / B=0.25$ and each figure is plotted with different initial kinetic energy $K / B=1.5,1,0.5,0$ as labeled on them.

with the Laplace transform,

$$
\tilde{\chi}^{(\mathrm{NO})}(s)=\frac{\beta}{\sin \left(\frac{\pi \alpha}{2}\right)}\left(\frac{s}{\Omega}\right)^{\alpha-1} .
$$

Figures 8 and 9 show the plot of the FMF Eq. (39) as a function of time and $\alpha$, respectively. Note that $\alpha=1$ recovers the normal Markovian FMF. For super-Ohmic case $\alpha>1$, the FMF Eq. (39) is negative and approaches $-\infty$ as $t \rightarrow 0$, but from Eq. (37) it is seen that $\chi^{(\mathrm{NO})}(0) \rightarrow+\infty$. Furthermore, these divergences in the super-Ohmic case are such that the static friction is vanishing, $\beta^{(0)}=\tilde{\chi}^{(\mathrm{NO})}(0)=\int_{0}^{\infty} \chi^{(\mathrm{NO})}(t) d t=0$. On the other hand, for sub-Ohmic diffusion $\alpha<1$ there is a divergence to $+\infty$ as $t \rightarrow 0$, hence the static friction is divergent, $\beta^{(0)}=+\infty$. This behavior is completely different from the Ohmic dissipative systems which have static frictions that are simply equal to the friction coefficient $\beta$.

A force-free system coupled to a bath with non-Ohmic spectral density of the form Eq. (38) exhibits anomalous diffusion [40] which is characterized by the mean square displace- 


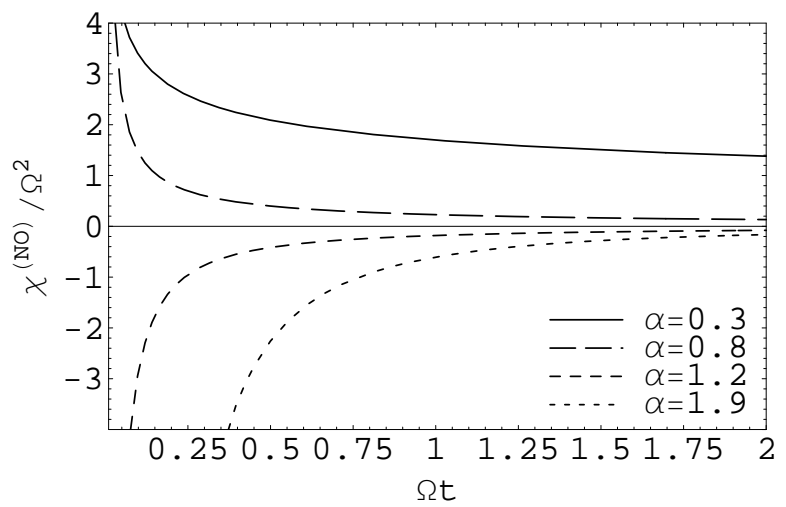

FIG. 8: The FMF Eq.(39) is plotted versus time $\Omega t$ for different values of $\alpha$. The friction coefficient is taken as $\beta / \Omega=1$.

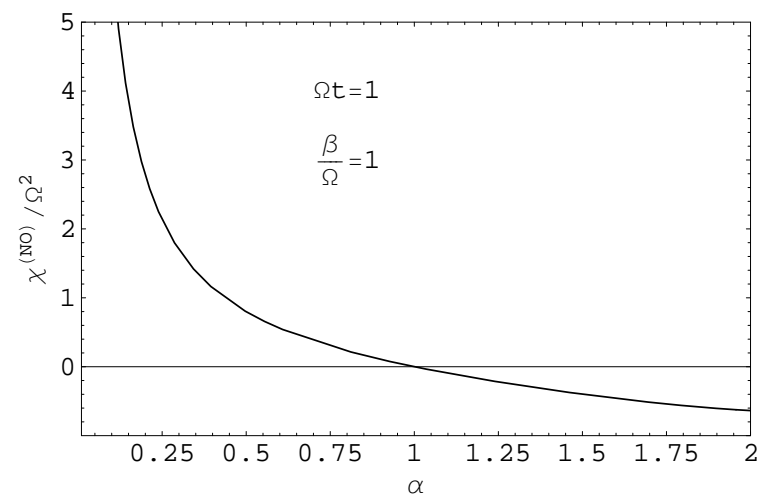

FIG. 9: The FMF Eq.(39) is plotted versus the parameter $\alpha$. The time and friction coefficient are taken as $\Omega t=1$ and $\beta / \Omega=1$, respectively.

ment given by

$$
\left\langle x^{2}(t)\right\rangle \sim t^{\alpha} \quad(t \rightarrow \infty)
$$

where, for $0<\alpha<1$ the system is called subdiffusive and for $1<\alpha<2$ the system is superdiffusive. The static friction can be used to distinguish between the sub-Ohmic $\left(\beta^{(0)} \rightarrow \infty\right)$, Ohmic $\left(\beta^{(0)} \rightarrow\right.$ finite $)$, and super-Ohmic $\left(\beta^{(0)} \rightarrow 0\right)$ environments which mean sub-diffusion, normal diffusion, super-diffusion for force-free systems.

Our expressions for the over-passing probability are valid for non-Ohmic dissipation as well, since the characteristic function Eq. (8) again has only one positive root. The Figure 10 shows the plot of the normalized root $y$ of Eq. (8) versus the parameters $\alpha$. The corresponding probabilities are indicated in Figure 11. The effective friction $\beta_{\text {eff }}$ is enhanced 


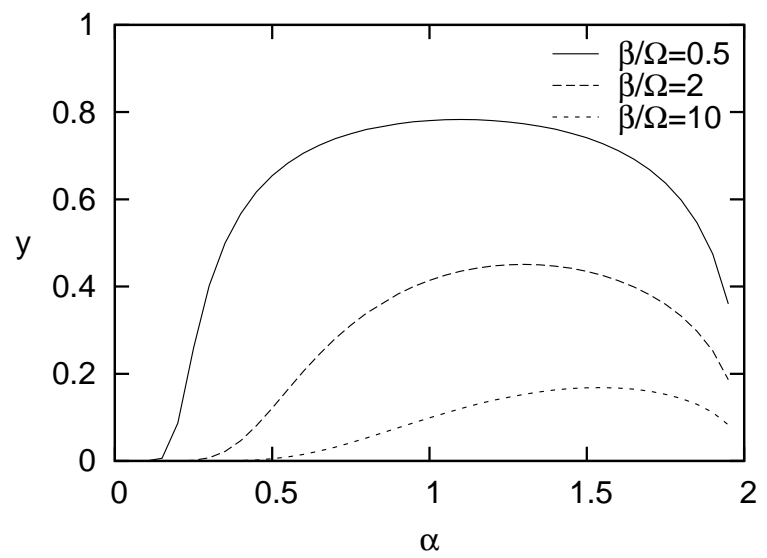

FIG. 10: The normalized root is plotted versus the parameter $\alpha$.

for the very subdiffusive or very superdiffusive systems.

We emphasize that our study is limited only to Gaussian distributions and hence does not include systems exhibiting non-Gaussian anomalous diffusion like Lévy flights.

\section{Quantum effects}

The previous three sections are dealing with the effects of the FMF and hence of the normalized root $y$ on the probability. Here, we investigate the effects of quantum noise on the dynamics. For this purpose we consider the exponential FMF Eq. (30) with the corresponding root Eq. (31). The over-passing probability is obtained by substituting this root and the Fourier transform of the FMF into Eq. (26). In order to compare our results with some previous studies we consider the fusion reaction of ${ }^{48} \mathrm{Ca}$ and ${ }^{238} \mathrm{U}$ nuclei with the same parameter set $[9,10,11]$. The friction coefficient is taken as $\beta / \Omega=3.29$, the memory time is $\Omega \tau=1 / 15$, the curvature parameter of the conditional saddle is $\Omega=1$ and the barrier height with respect to the initial position is $B=4$, in arbitrary units. The comparison of the probabilities for the classical and quantum systems is shown in Figure 12 which is in good agreement with the previous studies [9, 10, 11]. At low temperatures, the over-passing probability is enhanced since the variance of the position is larger when the quantum effects are included. At high temperatures, the classical over-passing probability is recovered. 

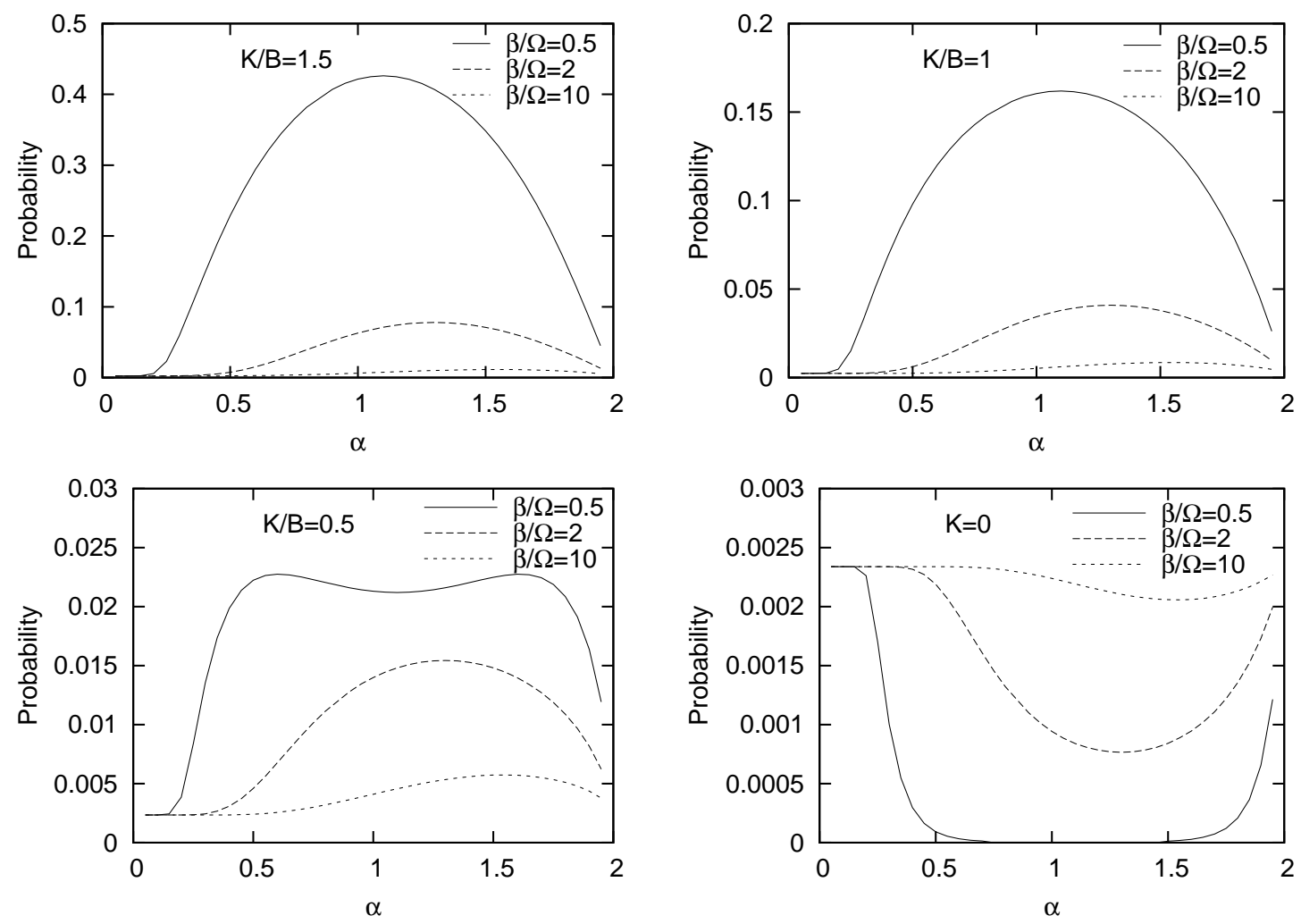

FIG. 11: The probability Eq. (15) for the non-Ohmic FMF Eq. (38) is plotted versus the friction $\alpha$. The temperature is taken so that $T / B=0.25$ and each figure is plotted with different initial kinetic energy $K / B=1.5,1,0.5,0$ as labeled on them.

\section{CONCLUSION}

The probability of a system to diffuse over a barrier is an important quantity in many research subjects such as activation processes in chemical physics, fusion and fission reactions as well as giant resonances in nuclear physics. In this work, we consider the evolution of a single-relevant variable according to the classical and quantal GLE. When the potential barrier has the shape of an inverted parabola, the asymptotic value of the over-passing probability is given by the complimentary error function according to Eq. (11). We show that in the case of classical GLE the asymptotic value of the over-passing probability is determined by a single dominant root $y=s_{1} / \Omega$ of the characteristic function $D\left(s_{1}\right)=0$, and given by a simple expression Eq. (15). The details of dissipation mechanism and memory effects enter into the expression only through the dominant root of the characteristic equation. One of the results we found is that for the initial kinetic energies $K$ less than the 


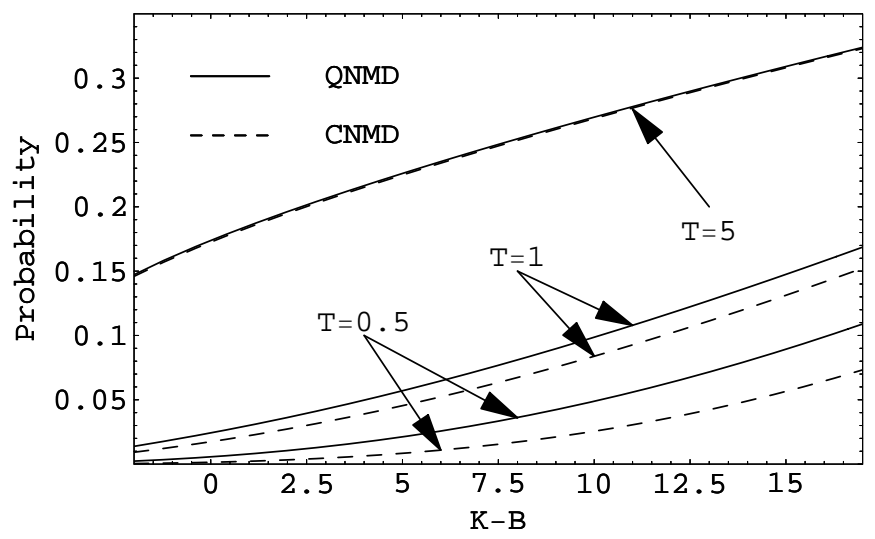

FIG. 12: The probabilities for the quantum and classical systems are plotted versus the initial kinetic energy $K$ with respect to the barrier height $B$ for the temperatures $T=0.5,1,5$. The quantum non-Markovian and the classical non-Markovian diffusions are indicated by solid and dashed lines, respectively.

barrier height $B$, which is the case for many physical situations, the over-passing probability of the diffusion due to the thermal fluctuations becomes maximum when the dominant root fits the condition given by Eq. (19). This is a result of the competition between dissipation and fluctuation. In the case of quantal GLE, the asymptotic value of the over-passing probability has the same structure as the classical one, except it involves a quantity which is determined by a numerical integration over the spectral density. The probability is enhanced at low temperatures where the quantum effects are relevant. The expression for the overpassing probability, Eq. (15) in the classical limit and Eq. (26) in quantal framework, are valid for a general FMF provided that the FMF has a well defined Laplace transform.

It is shown that the oscillatory behavior of the FMF can have an important impact on the factor $y$ and hence on the over-passing probability. For oscillation frequencies $\lambda$ less than the critical value Eq. (35), the non-Markovian dissipation $\beta_{\text {eff }}$ is reduced with respect to the Markovian one $\beta$. Whereas for frequencies exceeding the the critical value, the nonMarkovian dissipation is enhanced.

Our formulation also covers systems exhibiting anomalous diffusion with Gaussian noises. In this case, the static friction coefficient $\beta^{(0)}$ is zero or infinity for superdiffusive or subdiffusive systems, respectively. The feature allows to distinguish easily between these anomalies. The effective friction $\beta_{\text {eff }}$ is enhanced for subdiffusive systems $\alpha<1$ with respect to the 
Markovian friction coefficient $\beta$. Whereas there is a minimum of the effective friction or similarly maximum of the non-Ohmic root $y_{\max }^{(\mathrm{NO})}$ which changes with $\beta / \Omega$ in the superdiffusive region $\alpha>1$.

\section{Acknowledgments}

S.A. gratefully acknowledges the Physics Department of Middle East Technical University, D.B. acknowledges RCNP of Osaka University, and B.Y. acknowledges GANIL, RCNP of Osaka University and Physics Department of Tohoku University for the warm hospitality extended to them during their visits. The authors also thank Noboru Takigawa, Denis Lacroix and Kouhei Washiyama for fruitful discussions. This work is supported in part by the US DOE Grant No. DE-FG05-89ER40530, in part by the BDP grant from Turkish Science and Technology Council (TUBITAK), and in part by the JSPS Core-to-Core Program for Exotic Femto Systems and by JSPS Grant No. 18540268.

[1] C. W. Gardiner, Quantum Noise, Springer-Verlag, Berlin, 1991.

[2] U. Weiss, Quantum Dissipative Systems, World Scientific, Singapore, 1999.

[3] Y. Abe, S. Ayik, P.-G. Reinhard, and E. Suraud, Phys. Rep. 275, 49 (1996).

[4] P. Hanggi, P. Talkner, and M. Borkovec, Rev. Mod. Phys. 62, 251 (1990).

[5] H. Grabert, P. Schramm and G. L. Ingold, Phys. Rep. 168, 115 (1988).

[6] I. Percival, Quantum State Diffusion, Cambridge, Cambridge, 1998.

[7] A. O. Caldeira and A. J. Leggett, Physica A 121, 587 (1983).

[8] H. Mori, Prog. Theor. Phys. 33, 423 (1965).

[9] N. Takigawa, S. Ayik, K. Washiyama, and S. Kimura, Phys. Rev. C 69, 054605 (2004).

[10] S. Ayik, B. Yilmaz, A. Gokalp, O. Yilmaz, and N. Takigawa, Phys. Rev. C 71, 054611 (2005).

[11] K. Washiyama, B. Yilmaz, S. Ayik, and N. Takigawa, AIP Conf. Proc. 853, 187 (2006); [e-print arXiv:nucl-th/0606001].

[12] H. Hofmann, Phys. Rep. 284, 137 (1997).

[13] C. Rummel and H. Hofmann, Nucl. Phys. A 727, 24 (2003).

[14] P. Grigolini and F. Marchesoni, Adv. Chem. Phys. 62, 29 (1985). 
[15] F. Amblard, A. C. Maggs, B. Yurke, A. N. Pargellis, and S. Leibler, Phys. Rev. Lett. 77, 4470 (1996).

[16] F. Sciortino and P. Tartaglia, Physica A 236, 140 (1997).

[17] Q. Gu, E. A. Schiff, S. Grebner, F. Wang, and R. Schwarz, Phys. Rev. Lett. 76, 3196 (1996).

[18] J. Stephenson, Physica A 222, 234 (1995).

[19] D. Boilley and Y. Lallouet, J. Stat. Phys. 125, 477 (2006).

[20] Y. Abe, D. Boilley, B. G. Giraud, and T. Wada, Phys. Rev. E 61, 1125 (2000).

[21] D. Boilley, Y. Abe, and J. D. Bao, Eur. Phys. J. A 18, 627 (2003).

[22] R. F. Grote and J. T. Hynes, J. Chem. Phys. 73, 2715 (1980).

[23] R. F. Grote and J. T. Hynes, J. Chem. Phys. 74, 4465 (1981).

[24] P. Hanggi and F. Mojtabai, Phys. Rev. A 26, 1168 (1982).

[25] B. Carmeli and A. Nitzan, Phys. Rev. A 29, 1481 (1984).

[26] Y. I. Dakhnovskii and A. A. Ovchinnikov, Phys. Lett. A 113, 147 (1985).

[27] E. Pollak, H. Grabert, and P. Hanggi, J. Chem. Phys. 91, 4073 (1989).

[28] P. Hanggi, J. Stat. Phys. 42, 105 (1986).

[29] D. J. Tannor and D. Kohen, J. Chem. Phys. 100, 4932 (1994); D. Kohen and D. J. Tannor, J. Chem. Phys. 103, 6013 (1995).

[30] D. Banerjee, B. C. Bag, S. K. Banik, and D. S. Ray, Phys. Rev. E 65, 021109 (2002).

[31] J. R. Senitzky, Phys. Rev. 119, 670 (1960).

[32] R. Zwanzig, J. Stat. Phys. 9, 215 (1973).

[33] G. W. Ford, J. T. Lewis, and R. F. O’Connell, Phys. Rev. A 37, 4419 (1988).

[34] H. Grabert, P. Schramm, and G.-L. Ingold, Phys. Rev. Lett. 58, 1285 (1987).

[35] A. J. Leggett et al., Rev. Mod. Phys. 59, 1 (1987).

[36] E. Lutz, Phys. Rev. E 64, 051106 (2001).

[37] J. D. Bao and Y. Z. Zhuo, Phys. Rev. C 67, 064606 (2003).

[38] J. D. Bao, J. Chem. Phys. 124, 114103 (2006).

[39] A. D. Vinales and M. A. Desposito, Phys. Rev. E 73, 016111 (2006).

[40] R. Morgado, F. A. Oliveira, G. G. Batrouni, and A. Hansen, Phys. Rev. Lett. 89, 100601 (2002). 